\title{
Análisis de conocimientos sobre aulas de comunicación y lenguaje de futuros/as maestros/as
}

\section{Analysis of pre-service teachers' knowledge about communication and language classrooms}

\author{
(iD) Irene Lacruz-Pérez \\ Universidad de Valencia (España) \\ (D) Pilar Sanz-Cervera \\ Universidad de Valencia (España) \\ (iD Gemma Pastor-Cerezuela \\ Universidad de Valencia (España) \\ (iD) Raúl Tárraga-Mínguez \\ Universidad de Valencia (España)
}

\section{Resumen}

Uno de los recursos regulados para la adecuada inclusión de alumnado con necesidades específicas de apoyo educativo (NEAE) en centros educativos ordinarios en la Comunidad Valenciana son las aulas de comunicación y lenguaje (CyL). El éxito de este recurso depende, entre otros aspectos, de los conocimientos que tenga el profesorado tanto generalista como especialista sobre su funcionamento. Es por ello que el objetivo del presente trabajo es analizar los conocimientos, los errores y las lagunas de los estudiantes de último curso de los grados de Maestro/a en Educación Infantil y de Maestro/a en Educación Primaria sobre el funcionamiento de estas aulas. Asimismo, se comparan los resultados obtenidos por los estudiantes de las menciones de Pedagogía Terapéutica (PT) y Audición y Lenguaje (AL) con el resto de menciones. Para este análisis se ha empleado un instrumento de evaluación de tipo cuantitativo. Los resultados indican un menor conocimiento de los futuros maestros generalistas en comparación a los futuros maestros de educación especial, lo cual supone implicaciones poco favorables para la inclusión educativa. Los resultados sugieren la necesidad de ofrecer una formación más amplia e igualitaria para todos los grados y menciones en contenidos relacionados con la educación especial para garantizar prácticas inclusivas de calidad en las escuelas.

\begin{abstract}
One of the main resources to promote the inclusion of students with special educational needs in Valencian Community regular schools are the communication and language classrooms. The success of this resource depends, among other aspects, on the knowledge that both regular and special educational teachers have about its functioning. For this reason, the aim of this work is to analyze the knowledge, misconceptions, and gaps about the functioning of these classrooms that early childhood education and primary education pre-service teachers have in their final year at university. Likewise, the results obtained by special educational pre-service teachers are compared to the results obtained by regular pre-service teachers. To do this analysis, a quantitative assessment instrument has been used. Results indicate a lower knowledge of regular pre-service teachers compared to special educational pre-service teachers, which leads to unfavorable implications for educational inclusion. The results suggest the need to offer a broader and more equal training related to special education for all pre-service teachers in order to guarantee inclusive quality practices in schools.
\end{abstract}

\section{Palabras clave / Keywords}

Aulas de comunicación y lenguaje; educación inclusiva; formación de docentes; futuros maestros de educación infantil; futuros maestros de educación primaria.

Communication and language classrooms; inclusive education; teacher education; early childhood pre-service teachers; primary education pre-service teachers. 


\section{Introducción}

\subsection{Las aulas de comunicación y lenguaje como recurso de inclusión educativa}

Las aulas de comunicación y lenguaje (aulas CyL) son unidades específicas de educación especial ubicadas en centros educativos ordinarios de la Comunidad Valenciana, que escolarizan estudiantes con necesidades educativas especiales (NEE) derivadas del trastorno del espectro autista (TEA) y la discapacidad intelectual grave y profunda (o moderada en educación secundaria). En ellas suele haber entre 5 y 8 alumnos que trabajan con un educador, un especialista en pedagogía terapéutica (PT) y un especialista en audición y lenguaje $(\mathrm{AL})$. Se trata de aulas de características análogas a las de otras comunidades autónomas, como las aulas abiertas, aulas específicas o aulas de transición (véase la revisión de este tipo de aulas realizada por Martínez, Porto y Garrido, 2019), pero con la particularidad de que las aulas CyL están específicamente orientadas al trabajo relacionado con la comunicación y el lenguaje.

La creación de las aulas CyL se sitúa dentro de un marco de políticas impulsadas en favor de la educación inclusiva en diferentes países europeos, mediante las cuales se han integrado recursos materiales y personales de educación especial en centros educativos ordinarios (European Agency for Special Needs and Inclusive Education, 2019a).

De este modo, el sistema educativo valenciano, mediante la Orden 20/2019, establece que el alumnado escolarizado en las aulas CyL debe disponer de un grupo de referencia en el aula ordinaria próximo a su edad cronológica, así como participar en el mayor número posible de actividades planificadas en dicho grupo y para todo el alumnado del centro.

Esta modalidad de escolarización es, por tanto, un recurso facilitador de la educación inclusiva, entendida como la búsqueda de los mayores niveles de presencia, participación y aprendizaje en el sistema educativo de todo estudiante que se encuentre en riesgo de exclusión social (Ainscow, 2005). Esto implica que la presencia en un centro educativo ordinario del alumnado con necesidades específicas de apoyo educativo (NEAE) debe llevar consigo unas experiencias de calidad que le permitan lograr un aprendizaje adecuado en diferentes ámbitos, más allá de los contenidos curriculares.

Algunas investigaciones sobre el estudio de las aulas CyL apuntan que la experiencia de estas unidades es viable y alcanza resultados positivos en el aprendizaje del alumnado, siempre y cuando se cuente con los recursos necesarios para realizar una intervención individualizada y una adaptación metodológica adecuada (Tomás y Grau, 2016). Sin embargo, su función como recurso de inclusión educativa del alumnado con TEA o discapacidad intelectual es compleja y está condicionada por diferentes variables relacionadas con el profesorado.

Entre estas variables destacan las actitudes del profesorado hacia la inclusión (Lee, Yeung, Tracey y Barker, 2015; Van Mieghem, Verschueren, Petry y Struyf, 2018); su capacidad de cooperación y colaboración con el resto de profesionales de la educación (Hamilton- Jones y Vail, 2014; Shepherd et al., 2016); y sus conocimientos sobre la temática (Berry, 2011; de Boer, Pijl y Minnaert, 2011). Dada su importancia, en los últimos años estos tres aspectos han sido temas recurrentes en la investigación sobre educación inclusiva.

\subsection{Actitudes del profesorado hacia la educación inclusiva}

En 2011, de Boer et al. revisaron 26 estudios que habían analizado las actitudes del profesorado hacia la inclusión del alumnado con NEE, y encontraron, en términos generales, actitudes neutras o negativas. Asimismo, identificaron diversas variables que influyen en tales actitudes, como son la formación o la experiencia trabajando con alumnado con NEAE, siendo más positivas las actitudes cuanto mayor eran estas variables. Otro factor influyente es el tipo de diagnóstico, pues el profesorado se mostraba más proclive a la inclusión del alumnado con diversidad funcional de tipo física o sensorial que cognitiva o conductual. Unos años más tarde, Van Mieghem et al. (2018) llevaron a cabo otra revisión bibliográfica, en la que alcanzaron conclusiones similares a las de de Boer et al. (2011), ya que también identificaron estos tres mismos factores como altamente influyentes en las actitudes del profesorado.

Uno de los temas que presenta una relevancia singular en este ámbito es el de la comparación de las actitudes hacia la inclusión del profesorado generalista y el especialista de educación especial.

Los estudios que han analizado hasta ahora esta cuestión han encontrado que los especialistas de educación especial suelen mostrar actitudes más positivas hacia la inclusión educativa que los maestros generalistas (Woolfson, Grant y Campbell, 2007). Una de las principales ideas que refuerza esta realidad es la concepción tradicional del "rol del especialista" y el "rol del generalista" (Robinson, 2017). 
Forlin (2010), entre otros autores, explica que tradicionalmente se ha vinculado la "necesidad educativa especial" a la idea de que se requiere un tratamiento específico que los maestros generalistas no son capaces de proporcionar, lo que perpetúa la división entre educación ordinaria y educación especial, entorpeciendo de este modo el avance de los principios propios de la educación inclusiva.

No obstante, y afortunadamente, en las últimas décadas el papel del profesorado de educación especial ha evolucionado desde un enfoque clínico hacia uno más educativo, lo que ha motivado nuevas necesidades formativas tanto para maestros en activo como para futuros maestros (Shepherd et al., 2016). Entre ellas, destaca la urgencia de formar al profesorado en el paradigma de la cooperación y la "co-enseñanza", donde la educación del alumnado con NEAE es una responsabilidad compartida entre el profesorado generalista y el profesorado especialista (Fluijt, Bakker y Struyf, 2016; Hamilton-Jones y Vail, 2014). Esta es una idea fundamental para la educación inclusiva, pero que a su vez representa una gran complejidad.

\subsection{Cooperación y colaboración entre el profesorado}

En la investigación de Nilsen (2017), en la que se entrevistó a profesorado generalista y de educación especial en activo, se encontró que el nivel de coordinación y planificación conjunta entre ellos era muy limitado. Los generalistas se encargaban de diseñar el plan de trabajo adaptado para el aula ordinaria del alumnado con NEAE, mientras que los especialistas elaboraban el plan de atención individualizada, en la mayoría de casos sin el consenso necesario para evitar solapamientos.

Este mismo autor describió los factores principales que determinan, y a su vez dificultan, la puesta en práctica del paradigma de la cooperación: se precisa una estructura clara de trabajo, una buena planificación temporal, habilidades de trabajo en equipo, reflexión individual y una visión compartida sobre la educación del alumnado con NEAE, entre otros aspectos. Además, Nilsen (2017) concluyó que los especialistas consideraban que los maestros generalistas no tenían los conocimientos adecuados para participar en el diseño de los planes de atención individualizada.

\subsection{Conocimientos del profesorado sobre educación inclusiva}

La tercera variable de influencia en la educación inclusiva es en la que se centra la presente investigación: los conocimientos del profesorado. Entre ellos, algunos autores señalan la importancia del conocimiento sobre los diagnósticos de los estudiantes con NEAE que pueden estar escolarizados total o parcialmente en aulas ordinarias.

Estudios como el de Haimour y Obaidat (2013) han analizado los conocimientos del profesorado en activo sobre el TEA (el diagnóstico más habitual en las aulas CyL). En concreto, estos autores encontraron que los maestros de educación especial tenían un conocimiento más adecuado que los generalistas.

Un estudio similar fue el de Sanz Cervera, Fernández Andrés, Pastor Cerezuela y Tárraga Mínguez (2017), aunque se centraron en el conocimiento de futuros maestros. Entre sus resultados encontraron que los estudiantes del último año de los grados de Maestro/a en Educación Primaria y Educación Infantil tenían menor conocimiento y más dudas sobre el TEA que los estudiantes de las menciones de PT y AL.

Esta diferencia significativa entre el conocimiento de profesorado generalista y especialista queda avalada también por otros estudios como el de Flower, McKenna y Haring (2017), quienes encontraron que los estudiantes que cursaban la mención de educación especial presentaban mayor conocimiento que los futuros maestros generalistas sobre los tipos de problemas de conducta y las estrategias para su afrontamiento.

Este es un resultado negativo para la inclusión, ya que la falta de formación del profesorado generalista puede provocar un bajo sentimiento de autoeficacia en los maestros, altos niveles de estrés, la aplicación de métodos menos efectivos, una pobre gestión de la clase y, consecuentemente, un bajo rendimiento académico del alumnado y una inclusión educativa fallida (de Boer et al., 2011; Flower et al., 2017; Sanz- Cervera et al., 2017).

\subsection{Formación del profesorado e inclusión educativa}

Estas tres variables relacionadas con el profesorado, y por ende con el éxito de la educación inclusiva, son interdependientes y deben abordarse y mejorarse a través de la formación docente (de Boer et al., 2011; Lee et al., 2015; Vaz et al., 2015). Durante su formación, tanto los maestros generalistas como los de educación especial deberían desarrollar determinadas destrezas, adquirir conocimientos sobre los distintos tipos de diagnósticos, conocer los recursos de los que disponen y aprender técnicas de enseñanza, basadas en la 
investigación, que les permitan trabajar de forma efectiva con el alumnado con NEAE (Berry, 2011; Forlin y Chambers, 2011; Sanz- Cervera et al., 2017; Shepherd et al., 2017; Symeonidou, 2017; Van Mieghem et al., 2018; Vaz et al., 2015).

Igualmente, es necesario fomentar ya desde la formación inicial del profesorado una práctica reflexiva individual que permita a cada docente conocer su propia filosofía educativa y comprender la importancia y el sentido de la inclusión (Berry, 2011; Forlin y Chambers, 2011; Robinson, 2017; Symeonidou, 2017). Este es uno de los factores clave para que se produzca una cooperación real entre el profesorado, un aspecto que debe trabajarse en la formación mediante el desarrollo de habilidades y la adquisición de herramientas necesarias para trabajar en equipo (Berry, 2011; Hamilton- Jones y Vail, 2014; Robinson, 2017; Shepherd et al., 2017; Symeonidou, 2017).

Por último, es fundamental que todos estos aprendizajes vayan acompañados de una experiencia de campo que facilite el proceso de transición entre la formación teórica y el desempeño real como maestros inclusivos (Cardona- Moltó, Tichá y Abery, 2018; Forlin y Chambers, 2011; Robinson, 2017; Symeonidou, 2017).

La European Agency for Special Needs and Inclusive Education (2019b) resume los objetivos que debe perseguir la formación del profesorado en favor de la educación inclusiva en: (1) integrar los temas de "inclusión" y "diversidad" de forma transversal en la formación; (2) romper la distancia entre el rol de "maestro de educación especial" y "maestro generalista"; (3) fomentar la práctica reflexiva en torno a la cooperación necesaria entre todos los profesionales que trabajan en entornos inclusivos.

De acuerdo a estos principios, especialmente al dirigido a disminuir la separación entre los roles de docentes generalistas y especialistas en educación especial, el presente trabajo se orienta a analizar diferentes aspectos relacionados con la formación inicial del profesorado.

\subsection{Objetivos del estudio}

Los objetivos planteados en el presente estudio son:

1. Analizar el conocimiento de las aulas CyL de estudiantes de $4^{\circ}$ curso de los grados de Maestro/a de Educación Primaria y Maestro/a de Educación Infantil.

2. Comparar el conocimiento sobre las aulas CyL de estudiantes de $4^{\circ}$ curso del grado de Maestro/a de Educación Primaria de las menciones de PT y AL con el conocimiento del resto de estudiantes de los grados de Maestro/a (pertenecientes a las menciones de especialista en lengua extranjera, educación musical, ciencias y matemáticas, tecnologías de la información y la comunicación y al grado de Educación Infantil).

Consideramos que estos objetivos son relevantes ya que permitirán conocer hasta qué punto los futuros maestros son conscientes del funcionamiento de las aulas CyL, un conocimiento que es clave para poder llevar a cabo prácticas educativas verdaderamente inclusivas. Además, los resultados obtenidos en el presente estudio pueden ser de utilidad para reorientar la formación inicial que se proporciona a los futuros maestros y maestras.

\section{Metodología}

\subsection{Participantes}

En el presente estudio participaron 420 estudiantes de $4^{\circ}$ curso de los grados de Maestro/a en Educación Infantil y de Maestro/a en Educación Primaria cuyos datos demográficos se muestran en la tabla 1.

\section{Tabla 1}

Datos demográficos de los participantes 
Género

Masculino

Femenino

Estudiantes del grado de Maestro/a en Educación Infantil

Estudiantes del grado de Maestro/a en Educación Primaria (mención de PT)

Estudiantes del grado de Maestro/a en Educación Primaria (mención de $\mathrm{AL})$

Estudiantes del grado de Maestro/a en Educación Primaria (resto de menciones)

\subsection{Instrumento}

En el presente estudio se aplicó un cuestionario de conocimientos sobre el funcionamiento de las aulas CyL de elaboración propia. En primer lugar, se elaboró un conjunto de ítems relacionados con la normativa y funcionamiento de las aulas CyL. Estos ítems se distribuyeron a cinco profesionales de las aulas CyL con al menos dos años de experiencia en estas aulas para que valoraran su contenido. El feedback de estos expertos sirvió para depurar el contenido del instrumento y dejarlo en su formato final de 12 ítems.

Cada ítem es una afirmación relacionada con las aulas CyL, siendo el contenido de 6 de ellas verdadero y el de las otras 6 falso (ver Anexo 1).

Las opciones de respuesta eran: verdadero, falso o no lo sé. Las respuestas fueron codificadas como:

- Conocimientos, en caso de que el estudiante respondiera correctamente.

- Errores conceptuales, en caso de que el estudiante respondiera de manera incorrecta.

- Lagunas, en caso de que el estudiante respondiera la opción no lo sé.

Este tipo de codificación permite diferenciar entre los errores conceptuales, es decir, los ítems en los que el estudiante cree que conoce la respuesta, pero no es así; y las lagunas, que se corresponden con los ítems en los que el estudiante manifiesta que no conoce la respuesta.

\subsection{Procedimiento}

Los autores del presente estudio contactaron con el profesorado de los estudiantes de cuarto curso del grado de Maestro/a de Educación Primaria y grado de Maestro/a de Educación Infantil de la Universidad de Valencia para poder administrar el cuestionario elaborado en sus respectivas aulas universitarias.

Una vez que los estudiantes dieron su consentimiento para participar voluntariamente en el estudio, los autores administraron el cuestionario de forma presencial y por escrito. Los participantes no obtuvieron ningún tipo de compensación y su participación fue voluntaria y desinteresada.

\subsection{Análisis de datos}

Los análisis de este estudio se llevaron a cabo mediante el paquete estadístico SPSS versión 26. Se realizó un análisis multivariado de varianza (MANOVA), así como las correspondientes pruebas univariadas y posthoc de comparaciones múltiples para comparar conocimientos, errores y lagunas entre los 4 grupos de participantes: estudiantes de educación infantil, de pedagogía terapéutica, de audición y lenguaje y de otras menciones de primaria. Se calcularon los tamaños del efecto utilizando los valores de $\eta_{p}{ }^{2}$, de acuerdo con Cohen (1988): $\eta_{p}^{2}<.06$, tamaño de efecto pequeño; $\eta_{p}^{2}$ entre .06 y .14, mediano; y $\eta_{p}^{2}>.14$, grande.

\section{Resultados}


EI MANOVA reveló diferencias estadísticamente significativas entre los estudiantes de $4^{0}$ curso de las diferentes menciones incluidas en el análisis (Lambda de Wilks $(\lambda)=.707, F_{(9,1007)}=17.130, p<.001, \eta_{p}{ }^{2}=.109$ ). La tabla 2 muestra la media y desviación típica de las puntuaciones en los conocimientos, errores y lagunas obtenidos por los cuatro grupos de participantes, así como los resultados de las pruebas univariadas. Los resultados evidencian diferencias estadísticamente significativas entre los grupos en los conocimientos y las lagunas.

Las pruebas post-hoc de comparaciones múltiples mostraron un patrón de resultados similar para las variables de conocimientos y lagunas: los grupos de AL y PT (es decir, los dos grupos de especialistas en educación especial), fueron quienes obtuvieron los mejores resultados (más conocimientos y menos lagunas), seguidos del grupo de El y, en último lugar, el grupo de EP, que fue el que obtuvo los peores resultados (menos conocimientos y más lagunas).

No se encontraron diferencias estadísticamente significativas entre los grupos en los errores cometidos. Los cuatro grupos de participantes cometieron una cantidad de errores similar, que en todos los casos era ligeramente superior a un error del total de 12 ítems del cuestionario.

\section{Tabla 2}

Medias, desviación típica y valores de F obtenidos en la comparación de conocimientos sobre aulas CyL entre los grupos de EI, EP, AL y PT.

\begin{tabular}{|c|c|c|c|c|c|c|c|}
\hline & $\begin{array}{c}\text { EI } \\
(n=219)\end{array}$ & $\begin{array}{c}\text { EP } \\
(n=126)\end{array}$ & $\begin{array}{c}A L \\
(n=37)\end{array}$ & $\begin{array}{c}\text { PT } \\
(n=38)\end{array}$ & $F_{(3,416)}$ & $\eta_{p}^{2}$ & $\begin{array}{l}\text { Diferencias entre } \\
\text { grupos }\end{array}$ \\
\hline Conocimientos & & & & & $50.37^{\star *}$ & .266 & $\mathrm{AL}, \mathrm{PT}>\mathrm{El}>\mathrm{EP}$ \\
\hline M & 4.79 & 3.63 & 8.00 & 7.79 & & & \\
\hline DT & 2.40 & 2.48 & 2.38 & 2.20 & & & \\
\hline Errores & & & & & .447 & .003 & \\
\hline M & 1.10 & 1.08 & 1.16 & 1.32 & & & - \\
\hline DT & 1.07 & 1.14 & 1.76 & 1.04 & & & \\
\hline Lagunas & & & & & $42.44^{\star \star}$ & .234 & $\mathrm{EP}>\mathrm{El}>\mathrm{PT}, \mathrm{AL}$ \\
\hline M & 4.97 & 6.23 & 1.97 & 2.47 & & & \\
\hline DT & 2.53 & 2.58 & 1.79 & 2.02 & & & \\
\hline
\end{tabular}

Nota: El= Educación Infantil; EP= Educación Primaria; AL= Audición y Lenguaje; PT= Pedagogía Terapéutica. ${ }^{* *} \mathrm{p}<.001$.

\section{Discusión}

El objetivo del presente estudio era analizar el conocimiento sobre las aulas CyL que tienen los estudiantes de último curso de los grados de Maestro/a, y comparar los conocimientos de los futuros maestros generalistas y los futuros maestros especialistas de educación especial de AL y PT.

Los resultados evidencian que los futuros maestros de educación especial tienen un mayor conocimiento sobre las aulas CyL, y menos dudas respecto a ellas que los futuros maestros generalistas. Asimismo, también se ha encontrado un conocimiento más acertado sobre estas aulas por parte de los futuros docentes de Educación Infantil en comparación con los de Primaria.

Estos hallazgos coinciden con los resultados obtenidos en investigaciones previas, en las que se ha comparado el conocimiento de estudiantes del grado de Maestro/a en Educación Primaria e Infantil con el de los estudiantes de la mención de educación especial sobre determinados diagnósticos, como el TEA (Sanz Cervera et al., 2017), o sobre determinadas estrategias de intervención con alumnado con NEAE (Flower et al., 2017). 
El desconocimiento del profesorado generalista de un recurso educativo e inclusivo para el alumnado con TEA o discapacidad intelectual como son las aulas CyL, y la diferencia de conocimiento hallada respecto al profesorado de educación especial, tiene implicaciones negativas relevantes para los procesos de inclusión educativa.

Por una parte, la formación de los docentes y los conocimientos de estos sobre educación especial e inclusiva están estrechamente relacionados con sus actitudes hacia la inclusión (de Boer et al., 2011; Van Mieghem et al., 2018; Vaz et al. 2015). Por tanto, esto podría conducir a pensar que los futuros maestros generalistas participantes en este estudio podrían desarrollar actitudes poco favorables hacia la inclusión educativa del alumnado con NEAE, debido a una formación inicial en materia de educación especial inadecuada e insuficiente, tal y como han señalado previamente otros estudios (Woolfson et al., 2007). Además, esta carencia formativa podría disminuir su sentimiento de autoeficacia y aumentar sus niveles de estrés y preocupación al abordar la educación de los estudiantes con NEAE (Forlin y Chambers, 2011).

Asimismo, los resultados evidencian una vez más la barrera existente entre el profesorado generalista y especialista, y parecen reificar la idea de una suerte de división del trabajo en la que el profesorado especialista "es el responsable" de los estudiantes con NEAE, mientras que el profesorado generalista debe centrar sus esfuerzos en el resto de estudiantes.

En este sentido, los resultados sugieren que, ya desde la formación inicial, el profesorado generalista (especialmente el de la etapa de educación primaria), parece desconocer en qué consiste un recurso como el de las aulas CyL, un espacio en que la coordinación entre generalistas y especialistas debería ser un pilar básico, y que debería contribuir a desterrar esa concepción naif de "división del trabajo" entre generalistas y especialistas, tal y como reclaman numerosas investigaciones (Hamilton- Jones y Vail, 2014; Nilsen, 2017).

En el contexto en el que se ha desarrollado este estudio, la Comunidad Valenciana, estos resultados son especialmente delicados si se tiene en cuenta la normativa más reciente en materia de inclusión en esta comunidad, en la cual se establece como prioridad escolarizar a todo el alumnado en centros ordinarios, proporcionando a los que tengan NEAE los recursos necesarios dentro de la modalidad ordinaria (Orden 20/2019). Es decir, la probabilidad de que el futuro profesorado generalista participante en este estudio vaya a trabajar con alumnado con NEAE en sus aulas ordinarias es elevada, por lo que deberían conocer los recursos de los que disponen para atenderles adecuadamente.

Un resultado esperanzador del presente estudio es que los futuros maestros de educación infantil, pese a que muestran conocimientos significativamente menores sobre las aulas CyL que los especialistas, parecen tener un mayor conocimiento sobre ellas que el futuro profesorado de educación primaria. Este resultado podría sugerir la existencia de una mayor sensibilidad hacia la inclusión por parte de los futuros docentes de educación infantil en comparación con los de educación primaria, tal y como se ha encontrado en otros estudios previos (Dimitrios, Pinelopi y Harilaos, 2018; Štemberger y Kiswarday, 2018). Sin embargo, se trata de un resultado que a priori no era esperado en el presente estudio, ya que la formación en materia de educación especial es básicamente la misma en el grado de Maestro/a en Educación Primaria y en Educación Infantil, por lo que consideramos que es necesario realizar estudios adicionales para identificar los motivos de la diferencia de resultados entre los futuros docentes de educación infantil y de educación primaria.

Los resultados del presente estudio tienen implicaciones para la formación inicial del profesorado. Estos sugieren que el actual modelo de formación inicial del profesorado, al menos el de la institución universitaria en la que se ha realizado el estudio, podría no estar acorde con los principios básicos de la educación inclusiva. Nuestro modelo de formación de maestros y maestras corresponde al que Nash y Norwich (2010) denominan modelo de múltiples vías, en el que, tras una formación genérica, común a todos los maestros, hay una formación específica de diferentes especialidades, entre las cuales se encuentra la educación especial.

Probablemente, este modelo de formación inicial contribuye a perpetuar la diferenciación de roles entre profesorado de educación especial y generalista (Robinson, 2017), legitimando una diferenciación de responsabilidades, donde la educación de los estudiantes con NEAE queda relegada al profesorado de PT y AL, por ser los expertos, y alejada en cierto modo del profesorado generalista, por ser los inexpertos.

Para revertir esta realidad poco favorecedora para la inclusión educativa, existen varias posibilidades. Una de ellas sería aumentar el contenido de educación especial e inclusiva en la formación inicial común de los estudiantes de los grados de Maestro/a, tal y como proponen algunos estudios previos como el de Vélez Calvo, Tárraga Mínguez, Fernández Andrés y Sanz Cervera (2016) o el de Cardona Moltó et al. (2018), quienes encontraron un peso insuficiente de la educación especial e inclusión educativa en los planes de estudio de los grados de Maestro/a en Educación Infantil y Primaria en España. 
Otra opción sería la que Nash y Norwich (2010) denominan modelo de una única vía, que implica reorientar la formación inicial del profesorado, desde el modelo actual hacia un modelo en que todos los maestros sean formados para trabajar con alumnado con NEAE en aulas ordinarias, una opción que requiere cambios estructurales y organizativos importantes. A este respecto, Young (2011) analizó un plan de formación universitario de EE.UU. que unifica la educación generalista y la educación especial e identificó como una de las exigencias principales la necesidad de reorganización y restructuración de la institución universitaria, siendo necesaria la creación de nuevas estructuras docentes orientadas específicamente a la formación en educación inclusiva.

La interpretación de los resultados del presente estudio debe llevarse a cabo teniendo en cuenta algunas limitaciones. Pese a que se ha contado con una muestra amplia, de 420 estudiantes, el trabajo se ha llevado a cabo únicamente en una universidad, por lo que los resultados no son necesariamente representativos de los estudiantes del resto de facultades del territorio nacional. Además, los resultados se han obtenido exclusivamente a través de un instrumento de tipo cuantitativo.

Dadas estas limitaciones, proponemos como futuras líneas de investigación: ampliar el estudio a más universidades, pues pese a que las aulas CyL son un recurso concreto de la Comunidad Valenciana, existen aulas análogas en otras comunidades autónomas que juegan igualmente un papel importante en la educación inclusiva; combinar el enfoque cuantitativo empleado en este estudio con uno cualitativo que enriquezca los resultados y la interpretación de estos; y finalmente, proponemos también combinar los resultados sobre los conocimientos de los futuros maestros sobre este recurso de educación especial con un análisis de su sentimiento de autoeficacia, una variable también estrechamente relacionada con la educación inclusiva.

\section{Conclusiones}

Los resultados hallados en este estudio nos permiten concluir que en el contexto analizado el futuro profesorado de educación especial se encuentra más preparado para la educación inclusiva que los futuros docentes generalistas (de educación primaria especialmente), a nivel de conocimiento teórico al menos.

Esto nos conduce a una segunda conclusión, que es que para la consecución de la educación inclusiva no es suficiente la implantación de recursos de educación especial en centros educativos ordinarios, como ocurre con las aulas CyL. De acuerdo con lo concluido por la European Agency of Special Educational Needs (2019a) en uno de sus últimos informes, lo que se precisa además de esto es un cambio en la concepción de los recursos de educación especial, comprendiendo que en ellos se ve involucrada toda la comunidad educativa y que no atañe únicamente a los especialistas. Este cambio de paradigma debe calar en la concepción de lo que significa ser docente en la escuela inclusiva. Por tanto, se trata de un cambio que debe ser impulsado desde la formación (inicial y continua) del profesorado.

Esta necesidad manifiesta de mejorar la formación del profesorado (en especial la formación inicial de los futuros maestros y maestras generalistas), es además de carácter urgente, teniendo en cuenta que, de acuerdo con los principios de la educación inclusiva, se espera que durante los próximos años aumente el número de estudiantes con algún tipo de NEE escolarizados en centros educativos ordinarios (y por tanto se reduzca el número de estudiantes escolarizados en centros de educación especial).

Ante esta realidad cambiante, la formación inicial del profesorado debe adaptarse con celeridad, abandonando definitivamente los modelos de formación separada entre profesorado generalista y especialista, y aumentando el tronco común de formación en el que la educación inclusiva sea considerada una responsabilidad de todos los educadores (independientemente de su especialidad), fomentando así ya desde la formación inicial una cultura de cooperación entre profesionales de diferentes ámbitos. De este modo, la formación inicial dejará de ser un obstáculo y pasará a convertirse en una de las herramientas más valiosas para alcanzar las metas y retos que nos plantean los principios de la educación inclusiva.

\section{Apoyos}

Este estudio ha recibido financiación de recursos humanos por parte de la Universidad de Valencia (Contratos para la formación de personal investigador de carácter predoctoral, en el marco del subprograma "Atracción de talento" 2019. Código de contrato UV-INV-PREDOC19F1-1010132).

\section{Referencias}

Ainscow, M. (2005). Developing inclusive education systems: what are the levers for change? Journal of educational change, 6 (2), 109-124. https://doi.org/10.1007/s10833-005-1298-4 
Berry, R. (2011). Voices of experience: general education teachers on teaching students with disabilities. International Journal of Inclusive Education, 15 (6), 627-648. https://doi.org/10.1080/13603110903278035

Cardona-Moltó, M, C., Tichá, R. y Abery, B. (2018). Education for diversity in initial teacher preparation programmes: a comparative international study. Journal of e-learning and Knowledge Society, 14 (2), $79-95$. https://doi.org/10.20368/1971-8829/1499

Cohen, J. (1988). Statistical power analysis for the behavioral sciences. Hillsdale, NJ: Erlbaum.

de Boer, A., Pijl, S. J., y Minnaert, A. (2011). Regular primary schoolteachers' attitudes towards inclusive education: A review of the literature. International journal of inclusive education, 15 (3), $331-353$. https://doi.org/10.1080/13603110903030089

Dimitrios, S., Pinelopi, R., \& Harilaos, Z. (2018). School teachers' attitudes toward inclusive education in Greece. European Journal of Special Education Research, 3(3), 182-194. https://doi.org/10.5281/zenodo.1250179

European Agency for Special Needs and Inclusive Education (2019a). Changing role of specialist provision in supporting inclusive education: Mapping specialist provision approaches in european countries. (S. Ebersold, M. Kyriazopoulou, A. Kefallinou and E. Rebollo Píriz, Eds.). Odense, Denmark. https://bit.ly/32UsROa

European Agency for Special Needs and Inclusive Education (2019b). Teacher professional learning for inclusion: Literature review. (A. De Vroey, S. Symeonidou and A. Watkins, Eds.). Odense, Denmark. https://bit.ly/32OrGQk Flower, A., McKenna, J. W., y Haring, C. D. (2017). Behavior and classroom management: Are teacher preparation programs really preparing our teachers? Preventing School Failure: Alternative Education for Children and Youth, 61(2), 163-169. https://doi.org/10.1080/1045988X.2016.1231109

Fluijt, D., Bakker, C., y Struyf, E. (2016). Team-reflection: The Missing Link in Co-teaching Teams. European Journal of Special Needs Education, 31 (2): 187-201. https://doi.org/10.1080/08856257.2015.1125690.

Forlin, C. (2010) Reframing teacher education for inclusion. C. Forlin (Ed.), Changing paradigms and innovative approaches: Teacher education for inclusion (pp. 3-12). Routledge.

Forlin, C., y Chambers, D. (2011). Teacher Preparation for Inclusive Education: Increasing Knowledge but Raising Concerns. Asia Pacific Journal of Teacher Education, 39 (1), 17-32. https://doi.org/10.1080/1359866X.2010.540850

Haimour, A. I., y Obaidat, Y. F. (2013). School teachers' knowledge about autism in Saudi Arabia. World Journal of Education, 3 (5), 45-56. https://doi.org/10.5430/ wje.v3n5p45

Hamilton-Jones, B. M., y Vail, C. O. (2014). Preparing Special Educators for Collaboration in the Classroom: Pre-Service Teachers' Beliefs and Perspectives. International Journal of Special Education, 29 (1), 76-86.

Lee, F. L. M., Yeung, A. S., Tracey, D., y Barker, K. (2015). Inclusion of children with special needs in early childhood education: What teacher characteristics matter. Topics in Early Childhood Special Education, 35 (2), $79-88$. https://doi.org/10.1177/027 1121414566014

Martínez, R., Porto, M., y Garrido, C. F. (2019). Aulas de educación especial en España: análisis comparado. Siglo Cero, 50(3), 89-120. https://doi.org/10.14201/scero 201950389120

Nash, T., y Norwich, B. (2010). The initial training of teachers to teach children with special educational needs: A national survey of English Post Graduate Certificate of Education programmes. Teaching and Teacher Education, 26 (7), 1471 1480. https://doi.org/10.1016/j.tate.2010.06.005

Nilsen, S. (2017). Special education and general education-coordinated or separated? A study of curriculum planning for pupils with special educational needs. International Journal of Inclusive Education, 21 (2), $205-217$. https://doi.org/10.1080/13603116.2016.1193564

Orden 20/2019, de 30 de abril, de la Conselleria de Educación, Investigación, Cultura y Deporte, por la cual se regula la organización de la respuesta educativa para la inclusión del alumnado en los centros docentes sostenidos con fondos públicos del sistema educativo valenciano. [2019/4442]

Robinson, D. (2017). Effective inclusive teacher education for special educational needs and disabilities: Some more thoughts on the way forward. Teaching and Teacher Education, 61, 164-178. https://doi.org/10.1016/j.tate.2016.09.007

Sanz-Cervera, P., Fernández-Andrés, M. I., Pastor-Cerezuela, G., y Tárraga-Mínguez, R. (2017). Pre-service teachers' knowledge, misconceptions and gaps about autism spectrum disorder. Teacher Education and Special Education, 40 (3), 212-224. https://doi.org/10.1177/0888406417700963

Shepherd, K. G., Fowler, S., McCormick, J., Wilson, C. L., y Morgan, D. (2016). The search for role clarity: Challenges and implications for special education teacher preparation. Teacher Education and Special Education, 39 (2), $83-97$. https://doi.org/10.1177/0888406416637904

Štemberger, T., y Kiswarday, V. R. (2018). Attitude towards inclusive education: the perspective of Slovenian preschool and primary school teachers. European Journal of Special Needs Education, 33 (1), $47-58$. https://doi.org/10.1080/08856257. 2017.1297573

Symeonidou, S. (2017). Initial teacher education for inclusion: a review of the literature. Disability \& Society, 32 (3), 401422. https://doi.org/10.1080/09687599.2017.129 8992

Tomás, R., y Grau, C. (2016). Modalidades de escolarización para el alumnado con trastornos del espectro autista. Revista Nacional e Internacional de Educación Inclusiva, 9 (2), 35-53.

Van Mieghem, A., Verschueren, K., Petry, K., y Struyf, E. (2018). An analysis of research on inclusive education: a systematic search and meta review. International Journal of Inclusive Education, 1- 15. https://doi.org/10.1080/13603116.2018.1482012 
Vaz, S., Wilson, N., Falkmer, M., Sim, A., Scott, M., Cordier, R., y Falkmer, T. (2015). Factors associated with primary school teachers' attitudes towards the inclusion of students with disabilities. PLoS One, 10 (8). https://doi.org/10.1371/journal.pone. 0137002

Vélez-Clavo, X., Tárraga-Mínguez, R., Fernández-Andrés, M. I., y Sanz-Cervera, P. (2017). Formación inicial de maestros en educación inclusiva: una comparación entre Ecuador y España. Revista de Educación Inclusiva, 9 (3), $75-94$.

Woolfson, L., Grant, E., y Campbell, L. (2007). A comparison of special, general and support teachers' controllability and stability attributions for children's difficulties in learning. Educational Psychology, 27 (2), $295-306$. https://doi.org/10.1080/014 43410601066826

Young, K. A. (2011). Institutional separation in schools of education: Understanding the functions of space in general and special education teacher preparation. Teaching and Teacher Education, 27 (2), $483-493$. https://doi.org/10.1016/j.tate.2010.10.001 


\section{Anexo}

\section{CONOCIMIENTOS SOBRE EL FUNCIONAMIENTO DE LAS AULAS CYL}

Rodee las palabras VERDADERO o FALSO según los conocimientos que posea sobre las aulas CyL. Por favor, no invente las respuestas. Si no está seguro, rodee las palabras NO LO SÉ.

\begin{tabular}{|c|c|c|c|c|}
\hline 1 & $\begin{array}{l}\text { En las aulas CyL se atiende a alumnos con Trastorno del Espectro } \\
\text { Autista (TEA) y con Trastorno por Déficit de Atención con o sin } \\
\text { Hiperactividad (TDAH) }\end{array}$ & VERDADERO & FALSO & $\begin{array}{l}\text { NO } \\
\text { LO } \\
\text { SÉ }\end{array}$ \\
\hline 2 & Las aulas CyL se incluyen en centros de Educación Especial & VERDADERO & FALSO & $\begin{array}{l}\text { NO } \\
\text { LO } \\
\text { SÉ }\end{array}$ \\
\hline 3 & Hay aulas CyL en algunos Institutos de Educación Secundaria & VERDADERO & FALSO & $\begin{array}{l}\text { NO } \\
\text { LO } \\
\text { SÉ }\end{array}$ \\
\hline 4 & En las aulas CyL se utiliza la metodología TEACHH & VERDADERO & FALSO & $\begin{array}{l}\text { NO } \\
\text { LO } \\
\text { SÉ }\end{array}$ \\
\hline 5 & $\begin{array}{l}\text { La escolarización de los alumnos en las aulas CyL se hace a } \\
\text { petición expresa de los padres }\end{array}$ & VERDADERO & FALSO & $\begin{array}{l}\text { NO } \\
\text { LO } \\
\text { SÉ }\end{array}$ \\
\hline 6 & La ratio en las aulas CyL es de 15 alumnos & VERDADERO & FALSO & $\begin{array}{l}\text { NO } \\
\text { LO } \\
\text { SÉ }\end{array}$ \\
\hline 7 & En las aulas CyL hay diversas modalidades de escolarización & VERDADERO & FALSO & $\begin{array}{l}\text { NO } \\
\text { LO } \\
\text { SÉ }\end{array}$ \\
\hline 8 & $\begin{array}{l}\text { En las aulas CyL solamente trabaja un maestro/a de Educación } \\
\text { Especial de la especialidad de Pedagogía Terapéutica }\end{array}$ & VERDADERO & FALSO & $\begin{array}{l}\text { NO } \\
\text { LO } \\
\text { SÉ }\end{array}$ \\
\hline 9 & $\begin{array}{l}\text { La metodología que se utiliza en las aulas CyL es la metodología } \\
\text { Bobath }\end{array}$ & VERDADERO & FALSO & $\begin{array}{l}\text { NO } \\
\text { LO } \\
\text { SÉ }\end{array}$ \\
\hline 10 & $\begin{array}{l}\text { La metodología que se utiliza en las aulas CyL es la inclusión del } \\
\text { alumnado que atienden }\end{array}$ & VERDADERO & FALSO & $\begin{array}{l}\text { NO } \\
\text { LO } \\
\text { SÉ }\end{array}$ \\
\hline 11 & $\begin{array}{l}\text { Los alumnos escolarizados en las aulas CyL pasan una parte del } \\
\text { horario lectivo en esta aula y otra parte del horario lectivo en el aula } \\
\text { ordinaria }\end{array}$ & VERDADERO & FALSO & $\begin{array}{l}\text { NO } \\
\text { LO } \\
\text { SÉ }\end{array}$ \\
\hline 12 & $\begin{array}{l}\text { En el aula CyL trabajan un maestro/a de Pedagogía Terapéutica, } \\
\text { un maestro/a de Audición y Lenguaje y un educador/a de } \\
\text { Educación Especial. }\end{array}$ & VERDADERO & FALSO & $\begin{array}{l}\text { NO } \\
\text { LO } \\
\text { SÉ }\end{array}$ \\
\hline
\end{tabular}

